\title{
A perturbative approach to a nonlinear advection-diffusion equation of particle transport
}

\author{
Dominik Walter $^{a, *}$ and Horst Fichtner, Yuri Litvinenkov ${ }^{a, b}$ \\ ${ }^{a}$ Ruhr-Universität-Bochum, \\ Universitätsstr. , Bochum, Germany \\ ${ }^{b}$ University of Waikato, Department of Mathematics, \\ P.B. 3105, Hamilton, New Zealand \\ E-mail: dw@tp4.rub.de
}

We explore analytical techniques for modeling the nonlinear cosmic ray transport in various astrophysical environments which is of significant current research interest. While nonlinearity is most often described by coupled equations for the dynamics of the thermal plasma and the cosmic ray transport or for the transport of the plasma waves and the cosmic rays, we study the case of a single but nonlinear advection-diffusion equation. The latter can be approximately solved analytically or semi-analytically, with the advantage that these solutions are easy to use and, thus, can facilitate a quantitative comparison to data. We present our previous work in a twofold manner. First, instead of employing an integral method to the case of pure nonlinear diffusion, we apply an expansion technique to the advection-diffusion equation. We use the technique systematically to analyze the effect of nonlinear diffusion for the cases of constant and spatially varying advection combined with time-varying source functions. Second, we extend the study from the one-dimensional, Cartesian geometry to the radially symmetric case, which allows us to treat more accurately the nonlinear diffusion problems on larger scales away from the source. The results are compared to numerical solutions, which are also extended to more complex situations.

$37^{\text {th }}$ International Cosmic Ray Conference (ICRC 2021)

July 12 th - 23rd, 2021

Online - Berlin, Germany

\footnotetext{
${ }^{*}$ Presenter
} 


\section{Introduction}

In recent years several authors have addressed the nonlinearity of the cosmic ray transport in various astrophysical environments. The latter comprise the interstellar medium(e.g., Ptuskin et al.(2008),Amato and Blasi (2018), Holcomb and Spitovsky (2019) [1-3]), supernova remnants (e.g., Ptuskin et al. (2013), Bykov et al. (2014), Perri et al. (2016), Diesing and Caprioli (2019), Nava et al. (2019) [4-8]), heliospheric shock acceleration (e.g., Lee et al. (2012) [9]), as well as modulation (e.g. Moloto et al. (2018), Shalchi (2018) [10, 11]).

Most often the nonlinearity is described by two or more coupled differential equations for the background plasma and its waves and the cosmic rays (e.g., Wiener et al. (2019)[12]). As an alternative to solving these numerically we have recently investigated a single nonlinear transport equation and techniques for analytical and semi analytical solutions ( Litvinenko et al. (2017), Litvinenko et al. (2019)[13, 14]). These alternatives, which are based on a single advection-diffusion equation with a diffusion coefficent depending on the (gradient of the) particle distribution function, not only complement the development of the more detailed numerical models but also may guide as well as help to test the latter. In Litvinenko et al. (2017)([13]) we have concentrated on the implications of the nonlinearity for the so-called anomalous transport in one-dimensional, Cartesian advective-diffusive systems. Furthermore in Litvinenko et al. (2019) ([14]) we have studied the nonlinearity in the presence of time-varying source functions and the absense of advection. Now we extend these analyses here to systems with radial symmetry and with time-dependent sources as well as non-vanishing advection, which are often of interest in astrophysics, for instance for the particle transport in the solar wind. The method that is applied in this paper will be able to deal with advection, as well as time dependent sources and therefore combines the strengths of our previous works, without having the restriction of earlier approaches. To highlight the broad applicability of the mathematical method, we discuss a number of additional models, including different geometries and nonlinear diffusion processes.

The diffusive part of the nonlinear transport equation reads

$$
\frac{\partial f}{\partial t}=\nabla[D(f) \nabla f]
$$

We argued in Litvinenko et al. (2017) ([13]) that this formulation can be specified using the work of Ptuskin et al. (2008) ([1]), so that the diffusive part can be framed as

$$
D(f)=D_{0}\left(\hat{f}_{0} / r_{0}\right)^{v}|\nabla f|^{-v}
$$

In the presented work we want to give an analytical approximation for an energy dependent diffusion advection equation of the form:

$$
f_{t}+V f_{x}=\left(D_{0}\left|f_{x}\right|^{v} f_{x}\right)_{x}+\frac{1}{3} \frac{d V}{d x} f_{s}+Q_{0}
$$

In this equation $s=\ln \left(\frac{p}{p_{0}}\right)$. 


\section{Analytical method}

The analytical method presented here bases on expanding the distribution function $f$ in the nonlinear parameter $v$. So we insert the expression

$$
f=f_{0}+v f_{1}+v^{2} f_{2}+\ldots
$$

into the transport equation 3. We sort the resulting terms in orders of $v$ and get the resulting set of equations:

$$
\begin{aligned}
\mathcal{L} f_{0} & =Q_{0} \\
\mathcal{L} f_{1} & =Q_{1} \\
\ldots & \\
\mathcal{L} f_{n}= & Q_{n} \\
\mathcal{L}= & \frac{\partial}{\partial t}+V \frac{\partial}{\partial x}-D_{0} \frac{\partial^{2}}{\partial x^{2}}-\frac{1}{3} \frac{d V}{d x} \frac{\partial}{\partial s} \\
Q_{1}= & -D_{0}\left(1+\ln \left|f_{0, x}\right|\right) f_{0, x x} \\
Q_{2}= & -D_{0}\left(\frac{f_{1, x}}{f_{0, x}}-\frac{1}{2} \ln ^{2}\left(\left|f_{0, x}\right|\right)-\ln \left(\left|f_{0, x}\right|\right)\right) f_{0, x x} \\
& -D_{0}\left(1+\ln \left(\left|f_{0, x}\right|\right)\right) f_{1, x x} \text { etc. }
\end{aligned}
$$

To solve this set of equations we use the fundamental solution $\Gamma$ for the linear operator $\mathcal{L}$, the solution to $f_{n}$ results from a convolution of $\Gamma$ and $Q_{n}$.

The fundamental solution for $\mathcal{L}$ reads as:

$$
\Gamma=\frac{1}{\sqrt{4 \pi D_{0} t}} \exp \left[-\frac{(x-V t)^{2}}{4 D_{0} t}\right]
$$

The convolution is done by integration:

$$
f_{n}(x, t)=\int \Gamma_{\text {cart }}\left(x-x_{0}, t-t_{0}\right) Q_{n}\left(x_{0}, t_{0}\right) d t_{0} d x_{0}
$$

We present a number of different solutions, for different values of $D_{0}, V$ etc, up to the second order in $v$. Throughout this presentation we take $V=$ const., in the paper on which this presentation is based on Walter et al. (2020) ([16]) we also take into account a decreasing velocity profile.

\section{Various scenarios}

Next to the basic method presented above, we also present a different geometry (spherical geometry) and a different kind of nonlinear diffusion. The spherical geometry is motivated by various astrophysical systems, the alternate nonlinear diffusion is motivated by a need for a more consistent form of the diffusion in the case $f_{x} \rightarrow 0$. 


\subsection{Spherical geometry}

For the spherical geometry we take a look at the following nonlinear equation:

$$
f_{t}=\frac{1}{r^{2}}\left(r^{2} D_{0}\left|f_{r}\right|^{-v} f_{r}\right)_{r}+Q_{0}
$$

By expanding this equation in the same way as before, we derive again a set of equations:

$$
\begin{aligned}
\mathcal{L}_{\mathrm{rad}}= & \frac{\partial}{\partial t}-D_{0} \frac{1}{r^{2}} \frac{\partial}{\partial r}\left(r^{2} \frac{\partial}{\partial r}\right) \\
\mathcal{L}_{\mathrm{rad}} f_{n}= & Q_{n} \\
Q_{1}= & -\left(1+\ln \left(\left|f_{0, r}\right|\right)\right) f_{0, r r}-\frac{1}{r} \ln \left(\left|f_{0, r}\right|\right) f_{0, r} \\
Q_{2}= & -\frac{2}{r} \ln \left(\left|f_{0, r}\right|\right) f_{1, r}-\frac{2}{r} f_{1, r}-\ln \left(\left|f_{0, r}\right|\right) f_{1, r r}-\frac{1}{f_{0, r}} f_{1, r} f_{0, r r} \\
& -f_{1, r r}+\ln \left(\left|f_{0, r}\right|\right) f_{0, r r}+\frac{\ln ^{2}\left(\left|f_{0, r}\right|\right)}{2} f_{0, r r}+\ln ^{2}\left|f_{0, r}\right| \frac{f_{0, r}}{r}
\end{aligned}
$$

The fundamental corresponding Greensfunction and Greensformula were taken from Webb and Gleeson (1977) ([15]) and only had to be slightly adjusted to fit our equations.

$$
\begin{aligned}
f(r, t) & =\frac{1}{r^{2}} \int_{0}^{t} d t^{\prime} \int_{0}^{\infty} d r^{\prime} r^{\prime 2} Q\left(r^{\prime}, t^{\prime}\right) G\left(r^{\prime}, r, t-t^{\prime}\right) \\
G\left(r^{\prime}, r, t-t^{\prime}\right) & =\frac{\frac{r}{r^{\prime}}}{2 \sqrt{\pi\left(t-t^{\prime}\right)}} \\
& \times\left(\exp \left(-\frac{\left(r^{\prime}-r\right)^{2}}{4\left(t-t^{\prime}\right)}\right)-\exp \left(-\frac{\left(r^{\prime}+r\right)^{2}}{4\left(t-t^{\prime}\right)}\right)\right)
\end{aligned}
$$

We again present a number of different approximations up to the second order in $v$.

\subsection{Alternate nonlinearity}

The diffusion coefficient of section 1 has the disadvantage of diverging for $v \neq 0$ and $f_{x}=0$. For numerical simulations this can be circumvented by taking a maximum value $D_{\max }$ for $D_{0}\left|f_{x}\right|^{-\nu}$. Alternatively we can take the diffusion coefficient of the form:

$$
D=\frac{\tilde{D}}{\left|f_{x}\right|^{v}+\lambda_{0}}
$$

In this formulation the parameters $\tilde{D}$ und $\lambda_{0}$ has to be chosen, that the two limiting cases $f_{x} \rightarrow$ $0 \Rightarrow D \rightarrow D_{\max }$ and $v \rightarrow 0 \Rightarrow D \rightarrow D_{0}$ are satisfied. Taking this formulation, expanding $f$ in the 
already known manner, we derive again a set of equations:

$$
\begin{aligned}
\mathcal{L}_{\text {cons }}= & \frac{\partial}{\partial t}+V \frac{\partial}{\partial x}-\frac{\tilde{D}}{1+\lambda_{0}} \frac{\partial^{2}}{\partial x^{2}} \\
\Gamma_{\text {cons }}= & \frac{1}{\sqrt{4 \pi \frac{\tilde{D}}{1+\lambda_{0}} t}} \exp \left[-\frac{(x-V t)^{2}}{4 \frac{\tilde{D}}{1+\lambda_{0}} t}\right] \\
Q_{1}= & -\tilde{D} \frac{1+\ln \left|f_{0, x}\right|}{\left(1+\lambda_{0}\right)^{2}} f_{0, x x}, \\
Q_{2}= & -\tilde{D} \frac{\ln \left|f_{0, x}\right|+1}{\left(1+\lambda_{0}\right)^{2}} f_{1, x x}+\tilde{D} \frac{\ln ^{2}\left|f_{0, x}\right|+2 \ln \left|f_{0, x}\right|}{\left(1+\lambda_{0}\right)^{3}} f_{0, x x} \\
& -\tilde{D} \frac{\frac{1}{2} \ln ^{2}\left|f_{0, x}\right|+\ln \left|f_{0, x}\right|}{\left(1+\lambda_{0}\right)^{2}} f_{0, x x}-\tilde{D} \frac{f_{1, x}}{\left(1+\lambda_{0}\right)^{2} f_{0, x}} f_{0, x x}
\end{aligned}
$$

We can solve this equations again, by convoluting the source terms with the given fundamental solution.

\section{Results,Discussion and Outlook}

We present a seminanalytical formula to derive solutions to a distinct form of nonlinear diffusion advection equations. We also present a selected number of results of scenarios of different geometries and implementations of the diffusion coefficient, comparing them to much more time intensive numerical results. We demonstrate The quality of the approximations is dependent on the paramter $v$. The results obtained for the monoenergetic transport equation can be used as the groundwork for shock acceleration and non-constant velocity profiles. For more examples and scenarios you can take a look at Walter et al.(2020, Phys.Pl.27,id.082901)([16]).

The analytical results obtained can be used in future works as a groundwork for transport nonlinear transport equations, taking them as a reference point. Furthermore it is an imporevement to our previous works on nonlinear diffusion, because it is able to deal with advection and non constant sources.

\section{References}

[1] Non-linear diffusion of cosmic rays Advanced Space Research

[2] E. Amato and P. Blasi Cosmic ray transport in the Galaxy: A review Advanced Space Research

[3] C. Holcomb and A. Spitkovsky On the Growth and Saturation of the Gyroresonant Streaming Instabilities ApJ

[4] V.Ptuskin and V.Zirakashvili and E. Seo Spectra of Cosmic-Ray Protons and Helium Produced in Supernova Remnants ApJ

[5] A. Bykov and D. Ellison and S. Osipov and A. Vladimirov Magnetic Field Amplification in Nonlinear Diffusive Shock Acceleration Including Resonant and Non-resonant Cosmic-Ray Driven Instabilities ApJ 
[6] S. Perri and E. Amato and G. Zimbardo Transport of relativistic electrons at shocks in shelltype supernova remnants: diffusive and superdiffusive regimes $\mathrm{A} \& \mathrm{~A}$

[7] R. Diesing and D. Caprioli Spectrum of Electrons Accelerated in Supernova Remnants Physical Review Letters

[8] L. Nava and S. Recchia and S. Gabici and A. Marcowith and L. Brahimi and V. Ptuskin Non-linear diffusion of cosmic rays escaping from supernova remnants - II. Hot ionized media Monthly Notices of the RAS

[9] M. Lee and R. Mewaldt and J. Giacalone Shock Acceleration of Ions in the Heliosphere Space Science Reviews

[10] K. Moloto and N. Engelbrecht and R. Burger A Simplified Ab Initio Cosmic-ray Modulation Model with Simulated Time Dependence and Predictive Capability ApJ

[11] A. Shalchi Analytical Description of the Time-dependent Perpendicular Transport of Energetic Particles ApJ

[12] J. Wiener and E. Zweibel and M. Ruszkowski Cosmic ray acceleration of cool clouds in the circumgalactic medium Monthly Notices of the RAS

[13] Y. Litvinenko and H. Fichtner and D. Walter Anomalous Transport of Cosmic Rays in a Nonlinear Diffusion Model ApJ

[14] Y. Litvinenko and D. Walter and H. Fichtner A nonlinear energetic particle diffusion model with a variable source AIP Advances

[15] G. Webb and L. Gleeson Green's Theorem and Green's Functions for the Steady-State CosmicRay Equation of Transport Astrophysics and Space Science

[16] D. Walter and H. Fichtner and Y. Litvinenko A perturbative approach to a nonlinear advectiondiffusion equation of particle transport Physics of Plasmas 